\title{
Factors affecting the output pulse flatness of the linear transformer driver cavity systems with 5th harmonics
}

\author{
V. M. Alexeenko, ${ }^{1,2}$ M. G. Mazarakis, ${ }^{3}$ A. A. Kim, ${ }^{1,2}$ S. S. Kondratiev, ${ }^{1}$ V. A. Sinebryukhov, ${ }^{1}$ \\ S. N. Volkov, ${ }^{1}$ M. E. Cuneo, ${ }^{3}$ M. L. Kiefer, ${ }^{3}$ J. J. Leckby, ${ }^{3}$ B. V. Oliver, ${ }^{3}$ and P. D. Maloney ${ }^{4}$ \\ ${ }^{1}$ Institute of High Current Electronics, Russian Academy of Sciences, Tomsk 634055, Russia \\ ${ }^{2}$ Tomsk Polytechnic University, Tomsk 634050, Russia \\ ${ }^{3}$ Sandia National Laboratories, Albuquerque, New Mexico 87185, USA \\ ${ }^{4}$ Tomsk State Pedagogical University, Tomsk 634041, Russia \\ (Received 1 March 2016; published 19 September 2016)
}

\begin{abstract}
We describe the study we have undertaken to evaluate the effect of component tolerances in obtaining a voltage output flat top for a linear transformer driver (LTD) cavity containing 3rd and 5th harmonic bricks [A. A. Kim et al., in Proc. IEEE Pulsed Power and Plasma Science PPPS2013 (San Francisco, California, USA, 2013), pp. 1354-1356.] and for 30 cavity voltage adder. Our goal was to define the necessary component value precision in order to obtain a voltage output flat top with no more than $\pm 0.5 \%$ amplitude variation.
\end{abstract}

DOI: 10.1103/PhysRevAccelBeams.19.090401

\section{INTRODUCTION}

This paper describes the study we have undertaken to evaluate the effect of component tolerances in obtaining a voltage output flat top for a single square pulse linear transformer driver (LTD) cavity with 5th harmonics [1] and a 30 cavity voltage adder with vacuum insulated output line. Our goal was to define the necessary component value precision in order to obtain a voltage output flat top with no more than $\pm 0.5 \%$ amplitude variation. The components considered were the cavity switches, the capacitors, and the total brick inductance.

In Sec. I we present the design and parameters of the discharge circuit of the LTD cavity with 5th harmonics. The design is based on General Atomics oil filled capacitors model $35490(100 \mathrm{kV}, 12 \mathrm{nF})$ in the 1 st harmonic (standard) bricks, $35492(100 \mathrm{kV}, 1.4 \mathrm{nF})$ in the 3rd harmonic (modified) bricks, and $35493(100 \mathrm{kV}, 0.5 \mathrm{nF})$ in the 5th harmonic bricks. All these capacitors have the same dimensions $(58 \times 150 \times$ $146 \mathrm{~mm}^{3}$ ), allowing us to assemble several different configurations inside the same size cavity. These bricks provide the possibility to produce the flat top output cavity pulse with the same nominal inductance for all bricks [1].

In Sec. II we describe the simulation procedure in OrCAD software [2], which is based on the PSPICE circuit code, the choice of optimal configuration of the LTD cavity with 5th harmonics and the choice of the value of its resistive load. We present the OrCAD model of the module consisting of 30 series LTD cavities, and note some special features of the statistical simulation in the PSPICE circuit code.

Published by the American Physical Society under the terms of the Creative Commons Attribution 3.0 License. Further distribution of this work must maintain attribution to the author(s) and the published article's title, journal citation, and DOI.
At the beginning of Sec. III we describe separately the influence of the tolerance of the inductance of the cavity bricks, the switch jitter and the tolerance of the capacitor capacity on the flatness of the output pulse. Then we describe their joint influence on the flatness of the output pulse of the LTD module, and indicate the conditions allowing to get the $\pm 0.5 \%$ flat output pulse.

The results of the study are summarized in Sec. IV.

\section{DESIGN AND PARAMETERS OF THE DISCHARGE CIRCUIT OF THE LTD CAVITY WITH 5TH HARMONICS}

As was mentioned in [1], to produce a flat top output pulse the LTD cavity should contain $s$ bricks generating the 1 st harmonic, $m$ bricks generating the 3rd harmonic, and $f$ bricks generating the 5 th harmonic, where the numbers $s, m$ and $f$ satisfy the condition

$$
\left\{\begin{array}{l}
0.5 \lesssim \frac{m}{s} \lesssim 0.7 \\
\frac{f}{s} \approx \frac{m}{s}-0.4
\end{array}\right.
$$

Also in [1] we noted that the diameter of the LTD cavity should be large enough to contain more than one brick generating the 5th harmonic. In this paper we assume that the total number of the bricks is $s+m+f=20$, then at $f=2$ or 3 only two configurations, $10 \mathrm{~S}+7 \mathrm{M}+3 \mathrm{~F}$ and $11 \mathrm{~S}+7 \mathrm{M}+2 \mathrm{~F}$, satisfy the condition (2.1).

Since all capacitors have the same dimensions, both of these configurations can be assembled inside the same size cavity, presented in Figs. 1 and 2. The outer diameter of this cavity is $1974 \mathrm{~mm}$, the inner $765 \mathrm{~mm}$. The length of the cavity along the axis of the output line is $219 \mathrm{~mm}$ that means the vacuum electrical length is

$$
\tau_{i}=0.73 \mathrm{~ns} .
$$




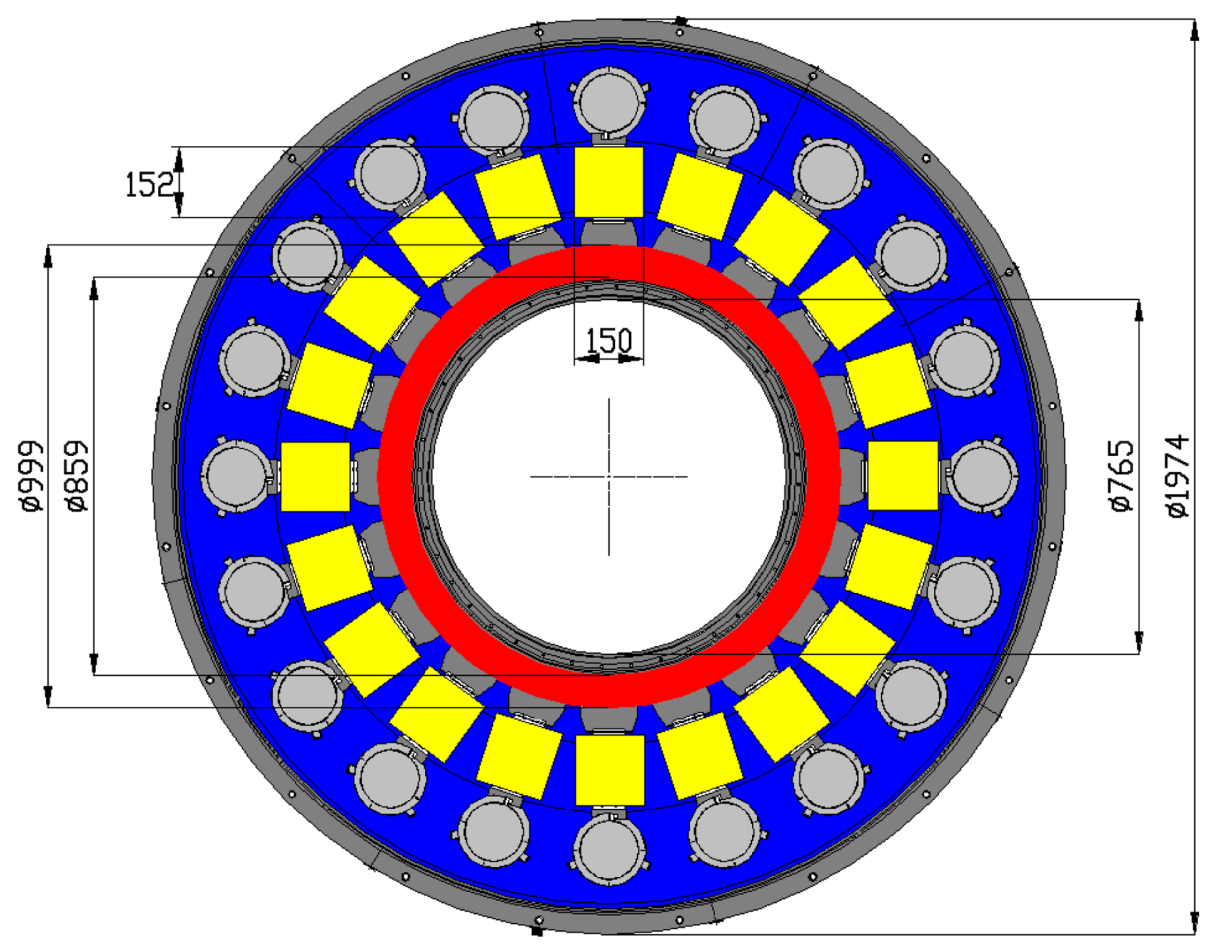

FIG. 1. Top view of the LTD cavity with 5 th harmonics and total number of bricks $s+m+f=20$. The dimensions here and in Fig. 2 are given in millimeters.

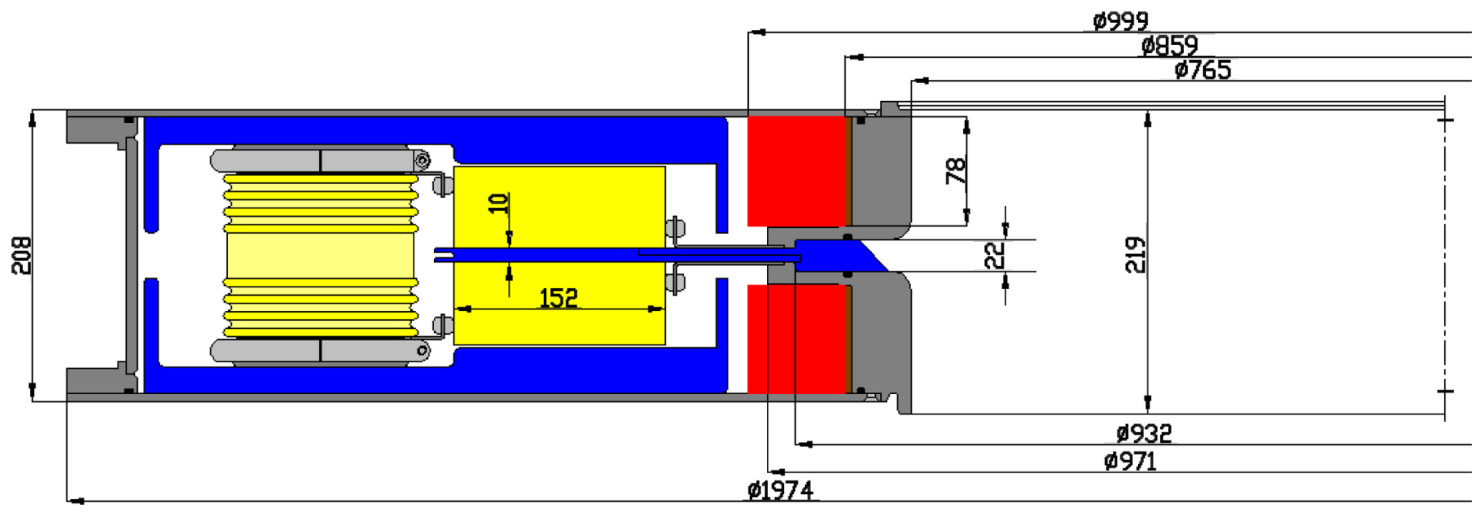

FIG. 2. Side view of the LTD cavity with 5 th harmonics and total number of the bricks $s+m+f=20$.

The areas indicated in red in Figs. 1 and 2 outline the volume occupied by the ferromagnetic cavity core. Assuming the ferromagnetic material fills $\sim 50 \%$ of this volume, the cross section of the core is $S_{C} \approx 4.5 \times 10^{-3} \mathrm{~m}^{2}$. The length of the center line of this volume is $l \approx 3 \mathrm{~m}$, by winding the core of 2605S3A magnetic alloy [3], whose specific resistivity and thickness of the tape are $\rho=13.8 \times 10^{-7} \mathrm{Ohmm}$ and $\delta=18 \times 10^{-6} \mathrm{~m}$, the equivalent resistance of the core for the rectangular voltage pulse will be [4]

$$
\text { Rcore }=8 \frac{\rho S_{C}}{\ell \delta^{2}} \approx 51 \mathrm{Ohm} .
$$

The value of the output inductance of this cavity is determined by the design of its insulator and the diameter of its output electrodes ( $\varnothing 971 \mathrm{~mm}$ in Fig. 2), that combine the outputs of the cavity bricks. In the design presented in Figs. 1 and 2, this inductance is

$$
\text { Lload }=0.95 \mathrm{nH} .
$$

\section{SIMULATION PROCEDURE IN ORCAD SOFTWARE}

\section{A. OrCAD models of the LTD cavity with 5th harmonics}

The OrCAD models of the LTD cavity with 5th harmonics used in this paper are almost the same as those used previously in [1]. Different are the values of the output inductance Lload $(=0.95 \mathrm{nH}$ now compared to $1.2 \mathrm{nH}$ in 


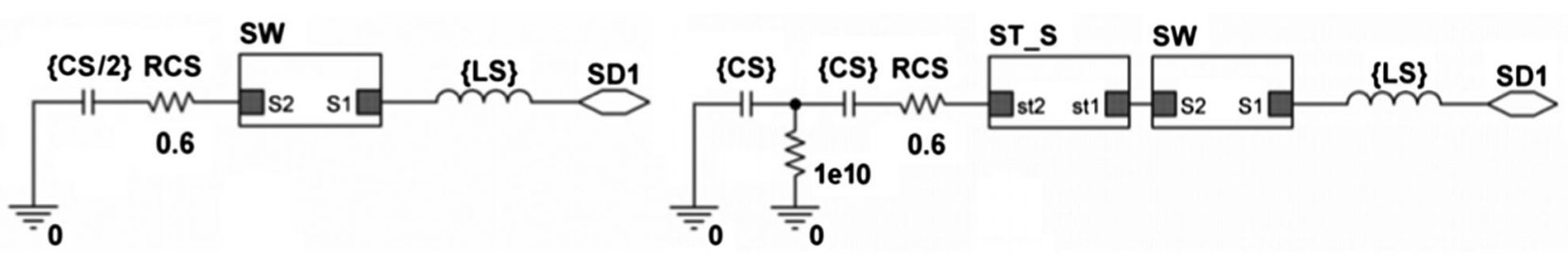

FIG. 3. The OrCAD models of the standard brick: on the left as presented in [1], and on the right as utilized in the present paper. The models of the $3 \mathrm{rd}$ and 5 th harmonic bricks were modified in the same way.

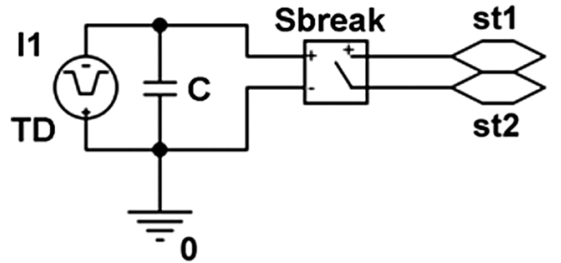

FIG. 4. The OrCAD model of the ST_S block.

[1]) and the resistance of the core Rcore ( $=51 \mathrm{Ohm}$ now compared to $1 \mathrm{MOhm}$ in [1]).

Another difference was introduced into the models of the cavity bricks (see Fig. 3 illustrating this difference in the models of the standard bricks, as example). Each capacitor is presented separately (CS instead of CS/2) to predict accurately the influence of the tolerance of the capacitor capacitance on the shape of the top of the output pulse, and the ST_S block is introduced to simulate the switch jitter.

The model of the ST_S block is shown in Fig. 4. It is similar to the one described in $[5,6]$. The ST_S block includes a "voltage-controlled switch breakout device" named Sbreak connecting the pins st 1 and st 2 to the rest of the circuit model at statistically varying times. The average time these pins are connected depends on the time parameter TD of the "pulse current source" I1. The time parameter TD is different for the different types of bricks, namely, for the standard bricks $\mathrm{TD}$ is set to TD $=10 \mathrm{~ns}$, while for the modified and 5 th harmonic bricks TD $=10 \mathrm{~ns}+t m$ and $\mathrm{TD}=10 \mathrm{~ns}+t m 2$ respectively. The values of $t m$ and $t m 2$ are the same in all modified and 5th harmonic bricks of the LTD cavity. However, they can be adjusted so the different type of bricks, standard and higher harmonic, could be fired at different times to adjust the output pulse shape.

\section{B. Optimal configuration of the LTD cavity with 5th harmonics and the value of the resistive load}

In the current paper we investigate the module consisting of $n=30$ series LTD cavities with 5th harmonics that operates in IVA mode. That means that the module load Rload is determined by each cavity load $R$ by the equation

$$
\text { Rload }=n \cdot R,
$$

the output line of this module consists of a series of coaxial sections (and is vacuum insulated), the impedance of these

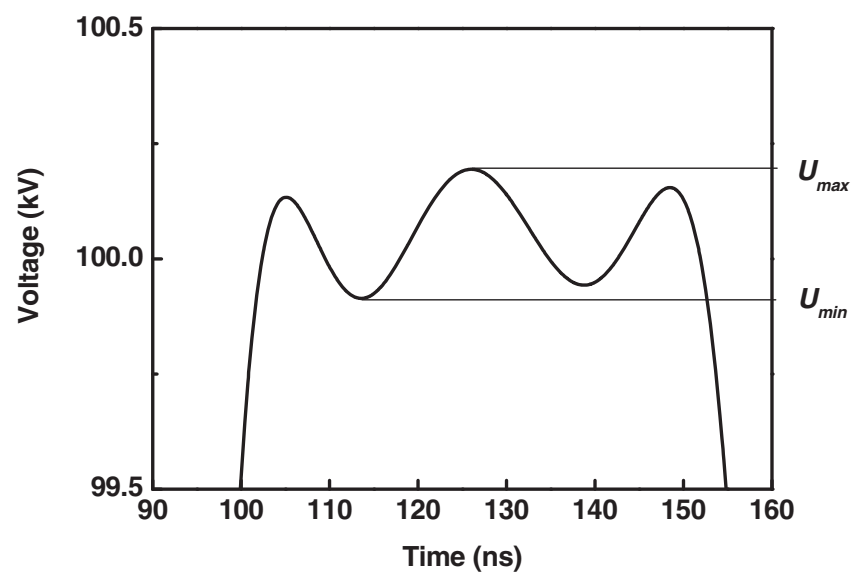

FIG. 5. Definition of the maximal $U_{\max }$ and minimal $U_{\min }$ amplitude of the voltage pulse across the resistive load $R$ in Eq. (3.2).

sections gradually increases from $R$ in the vicinity of the 1 st cavity to Rload in the vicinity of the 30th cavity, and the neighboring cavities fire with nominal delay $\tau_{i}$ one after the other.

Assuming the same tolerances of the module parameters and characteristics, such LTD module generates across the resistive load given by Eq. (3.1) the output pulse whose shape reproduces the shape of the pulse which generates each its cavity across the resistive load $R$. Therefore the choice of the optimal module load Rload was made by

TABLE I. Flatness of the top of the output pulse depending on the configuration of the LTD cavity with 5th harmonics, consisting of $s+m+f=20$ bricks at nominal parameters of these bricks.

\begin{tabular}{lcccc}
\hline \hline Configuration & $t m, \mathrm{~ns}$ & $t m 2, \mathrm{~ns}$ & $R$, Ohm & deltaU $/ 100 \mathrm{kV}, \%$ \\
\hline $10 \mathrm{~S}+7 \mathrm{M}+3 \mathrm{~F}$ & -0.5 & -0.6 & 0.88 & 2.06 \\
$11 \mathrm{~S}+7 \mathrm{M}+2 \mathrm{~F}$ & -0.6 & -1.3 & 0.79 & 0.48 \\
\hline \hline
\end{tabular}

Please note that $t m=-0.6 \mathrm{~ns}$ in Table I indicates that the switches in the 3rd harmonic bricks are closed $0.6 \mathrm{~ns}$ before the closure of the switches in the standard bricks. $t m 2=-1.3 \mathrm{~ns}$ indicates, the switches in the 5th harmonic bricks close $1.3 \mathrm{~ns}$ before those in the standard bricks. Positive values mean that these switches are closed after the closure of the standard brick switches. 

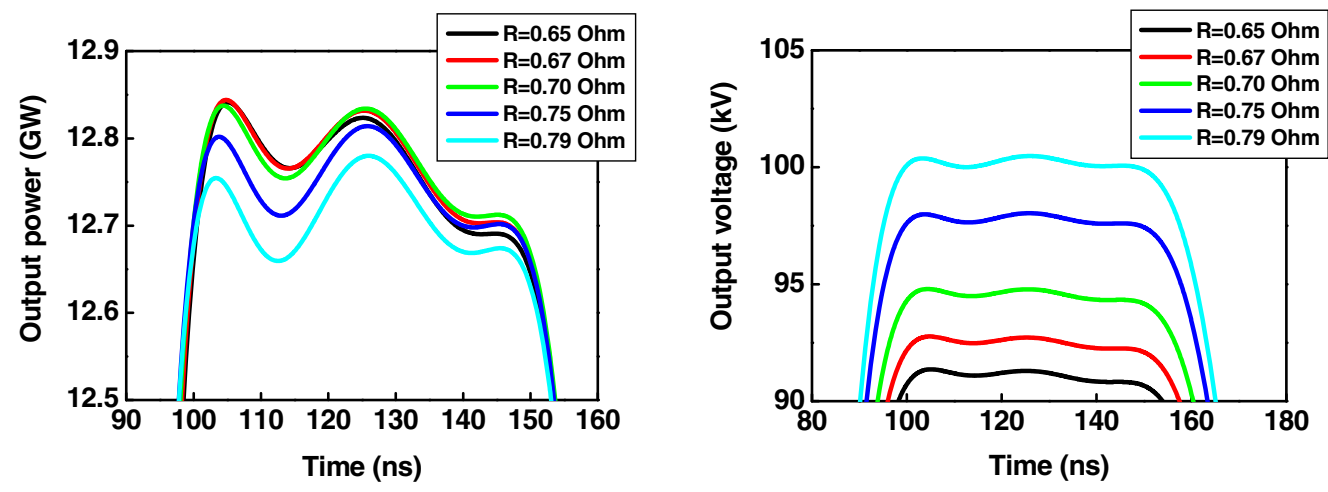

FIG. 6. Output power and voltage of the output pulse of the LTD cavity in $11 \mathrm{~S}+7 \mathrm{M}+2 \mathrm{~F}$ configuration depending on the resistance of the cavity load $R$.

choosing the optimal resistive load $R$ of only one LTD cavity with 5 th harmonics.

To choose the cavity load $R$ it was necessary to define, what configuration-10S $+7 \mathrm{M}+3 \mathrm{~F}$ or $11 \mathrm{~S}+7 \mathrm{M}+$ $2 \mathrm{~F}$ - generates the most flat output pulse. To answer this question, the shape of the output pulse depending on $R$ was simulated in both configurations at nominal capacitance of the storage capacitors, nominal inductance of the cavity bricks $(\mathrm{LS}=\mathrm{LM}=\mathrm{LF}=200 \mathrm{nH})$, and without any spread in the switch closing. The flatness of the top of the output pulse in these simulations was characterized by the parameter

$$
\operatorname{delta} U=U_{\max }-U_{\min },
$$

where $U_{\max }$ and $U_{\min }$ are maximal and minimal amplitude of the voltage pulse across the resistive load $R$ (see Fig. 5). The value of $R$ was varied with a step of $0.01 \mathrm{Ohm}$ to define what $R$ results in the mean output voltage of $\sim 100 \mathrm{kV}$ at $100 \mathrm{kV}$ charge voltage of the storage capacitors. Then the delays $\mathrm{tm}$ (in the bricks generating the $3 \mathrm{rd}$ harmonic) and tm2 (in the bricks generating the 5th harmonic) were determined resulting in the minimum possible deltaU in each configuration. ${ }^{1}$

Final results of this simulation are shown in Table I, which indicates that the most flat output pulse is generated by the $11 \mathrm{~S}+7 \mathrm{M}+2 \mathrm{~F}$ configuration. Note that in $10 \mathrm{~S}+$ $7 \mathrm{M}+3 \mathrm{~F}$ configuration the flatness of the top of the output pulse (at nominal parameters of the cavity bricks) is $\pm 1.03 \%$, which is larger by a factor of 2 from the required flatness of $\pm 0.5 \%$. In $11 \mathrm{~S}+7 \mathrm{M}+2 \mathrm{~F}$ configuration the

\footnotetext{
${ }^{1}$ In practice the needed values of the delays $t m$ and $t m 2$ can be provided, for example, by proper choice of the trigger resistance $R_{\text {tr }}$ in the standard, modified, and 5th harmonics bricks. The delay of the switch closing depends on this resistance as $t \approx R_{\mathrm{tr}} C_{\mathrm{tr}}$, where $C_{\text {tr }}$ is the capacitance of the switch trigger electrode to ground. At $C_{\mathrm{tr}} \approx 10 \mathrm{pF}$ [7] and $R_{\mathrm{tr}} \approx 500 \mathrm{Ohm}$ in the standard bricks, the delay $t m=-0.6$ ns (see bottom row in Table I) will require $R_{\mathrm{tr}} \approx 440 \mathrm{Ohm}$ in the modified bricks, and the delay $\operatorname{tm} 2=-1.3 \mathrm{~ns} R_{\mathrm{tr}} \approx 370 \mathrm{Ohm}$ in the 5th harmonics bricks.
}

flatness is $\pm 0.24 \%$, or $\sim 2$ times less than the required $\pm 0.5 \%$. Because of this reason in all next simulations described below, the configuration of the LTD cavity with 5 th harmonics is assumed to be $11 \mathrm{~S}+7 \mathrm{M}+2 \mathrm{~F}$.

Normally the pulse power systems are designed to provide the peak power output pulse. Figure 6 presents the simulated output power and voltage pulses of the cavity at nominal parameters in $11 \mathrm{~S}+7 \mathrm{M}+2 \mathrm{~F}$ configuration across the load resistance $R$, that predict the peak power of $\sim 12.84 \mathrm{GW}$ across the resistance $R=0.67 \mathrm{Ohm}$. But the output voltage of $\sim 100 \mathrm{kV}$ is expected at $R=0.79 \mathrm{Ohm}$, when the peak power is only $\sim 0.5 \%$ less than at $R=0.67 \mathrm{Ohm}$. Therefore in all the next simulations described below we assume the resistive cavity load being equal to

$$
R=0.79 \mathrm{Ohm},
$$

and corresponding 30 cavity module load being equal to

$$
\text { Rload }=23.7 \mathrm{Ohm} \text {. }
$$

At such $R$ and Rload the mean electrical field in the AK gap of the output line of the module is $\sim 200 \mathrm{kV} / \mathrm{cm}$ in the vicinity of the first cavity and $\sim 240 \mathrm{kV} / \mathrm{cm}$ in the vicinity of its 30th cavity.

\section{OrCAD model of the module consisting of 30 series LTD cavities with 5th harmonics}

The OrCAD model of the module consisting of 30 series LTD cavities with 5th harmonics is shown in Fig. 7.

In this model, the LTD cavities (Stage 1 to Stage 30) are connected via the sections of the output vacuum coaxial line, which are represented as LCL-circuits. ${ }^{2}$ The

\footnotetext{
${ }^{2}$ Such representation is due to the fact that when PSPICE is simulating the schematics with numerous short transmission line sections, the PSPICE model of the ideal transmission line may generate in these sections parasitic high voltage spikes of very small duration $\left(\sim 10^{-15} \mathrm{~s}\right)$ that lengthen largely each run and garble its results.
} 


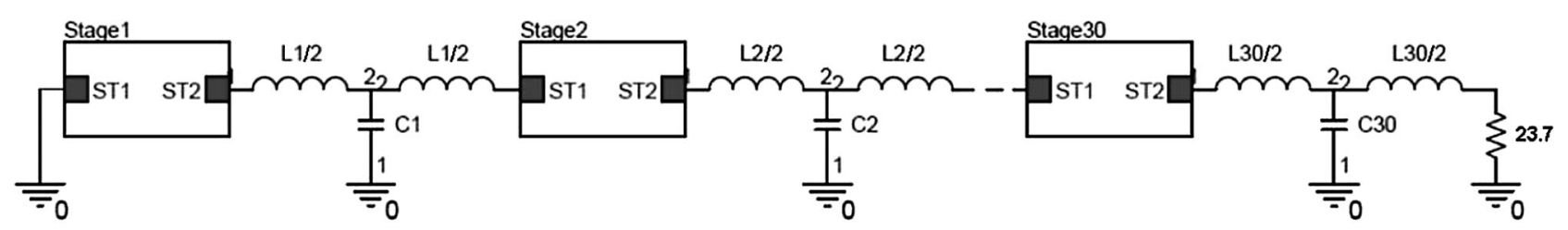

FIG. 7. The OrCAD model of the module consisting of 30 series LTD cavities with 5th harmonics terminated with a load of 23.7 Ohm.

transmission line with the impedance $\rho_{i}$ and electrical length $\tau_{i}$ is simulated as an LCL-circuit with $L_{i}$ and $C_{i}$ given by

$$
\left\{\begin{array}{l}
\mathrm{L}_{i}=\rho_{i} \tau_{i} \\
\mathrm{C}_{i}=\tau_{i} / \rho_{i} .
\end{array}\right.
$$

Since the geometric length of all line sections is the same and according to Fig. 2 equals to $219 \mathrm{~mm}$ (which is the axial length of the cavities), all $\tau_{i}$ are the same and equal to $\tau_{i}=0.73 \mathrm{~ns}$. According to the Eqs. (3.3) and (3.4), the impedance of these line sections increases with the step of $0.79 \mathrm{Ohm}$ from $\rho_{1}=0.79 \mathrm{Ohm}$ at the output of the $1 \mathrm{st}$ cavity to $\rho_{30}=23.7 \mathrm{Ohm}$ at the output of the 30th cavity. The module is connected to the resistive load of $23.7 \mathrm{Ohm}$. Since Rload $=\rho_{30}$, the length of the line between the 30th cavity and the load is of no concern (and is assumed to be the same $219 \mathrm{~mm}$ ).

\section{Some features of the statistical simulation in the PSPICE circuit code}

For statistical simulation, the PSPICE circuit code is using the Monte Carlo analyses and requires same type of distribution for all variable values of the parts in the OrCAD model. In [1], the statistical simulation of the switch jitter was performed by using the Gaussian distribution. The switch closing time was assumed to be equal to

$$
t_{s w}=\bar{t} \pm \sigma_{1 T},
$$

where $\bar{t}$ is the mean time the switch closes

$$
\bar{t}=\frac{1}{N} \sum_{i=1}^{N} t_{i},
$$

$\sigma_{1 T}$ is the standard deviation of the switch closing time (i.e., the switch jitter), equal to

$$
\sigma_{1 T}=\sqrt{\frac{1}{N} \sum_{i=1}^{N}\left(t_{i}-\bar{t}\right)^{2}},
$$

where $t_{i}$ is the time the switch closes in the ith shot of a series including $N$ shots; $t_{i}$ obeys Gaussian distribution.

Below we investigate the flatness of the top of the output pulse of an LTD cavity with 5th harmonics as well as an LTD module consisting of 30 such cavities (model presented in Fig. 7) depending on the switch jitter, the possible inaccuracy of the capacitance of the storage capacitors, and of the inductance of the cavity bricks. Because the PSPICE code requires the same type of distribution for all variable values, we assume that the capacitance of the capacitors and the inductance of the cavity bricks also obey Gaussian distribution.

According to General Atomics, the tolerance of the capacitance of the LTD capacitors is $\pm 10 \%$. Assuming that this tolerance is for all the capacitors, then the Gaussian distribution of the capacitance will have a standard deviation equal to $\sigma_{1 C} / \bar{C}=3.33 \%$. The tolerance of the inductance of the cavity bricks is not known; it may depend on the tolerance of the dimensions of the mechanical details as well as on the tolerance of the inductance of the capacitors and the switches. In the model we are using the standard deviation of the brick inductance in the form $\sigma_{1 L} / \bar{L}$, where $\bar{L}=200 \mathrm{nH}$ is the nominal inductance of the cavity bricks [1].

In the statistical simulation, the value of deltaU (see Eq. (3.2) and Fig. 5) varies in each of $N \geq 800$ iterations per solve. Then to describe the results of the solve we are using the parameter MDU (Maximum Delta $U$ ), which equals to the maximum deltaU observed in these $N$ iterations. By using this definition, the required flatness of the output pulse top of $\pm 0.5 \%$ becomes equivalent to the requirement $\mathrm{MDU} / U_{\mathrm{LOAD}}=1 \%$.

\section{SIMULATION RESULTS}

\section{A. Influence of the tolerance of the brick inductance on the flatness of the output pulse}

The influence of the tolerance of the brick inductance on the flatness of the output pulse is presented in Table II for one cavity loaded into $R=0.79 \mathrm{Ohm}$ load, and in Table III for the module of 30 cavities connected in series to the load Rload $=23.7 \mathrm{Ohm}$. These simulations were conducted at nominal capacitance of the brick capacitors generating the 1st, 3rd, and 5th harmonics and without switch jitter.

As appears from these tables that the tolerance of the brick inductance affects the flatness of the output pulse of the module much less than that of the cavity. This is

TABLE II. Flatness of the top of the output pulse of the LTD cavity depending on the tolerance of the brick inductance.

\begin{tabular}{llllll}
\hline \hline$\sigma_{1 L} / \bar{L}, \%$ & 0 & 0.833 & 1.67 & 2.5 & 3.33 \\
MDU $/ 100 \mathrm{kV}, \%$ & 0.48 & 0.93 & 1.5 & 2.09 & 2.77 \\
\hline \hline
\end{tabular}


TABLE III. Flatness of the top of the output pulse of the LTD module consisting of 30 cavities connected in series depending on the tolerance of the brick inductance.

\begin{tabular}{lllllll}
\hline \hline$\sigma_{1 L} / \bar{L}, \%$ & 0 & 0.833 & 1.67 & 2.5 & 3.33 & 3.58 \\
$\mathrm{MDU} / 3 \mathrm{MV}, \%$ & $0.47^{\mathrm{a}}$ & 0.58 & 0.67 & 0.82 & 0.96 & 1 \\
\hline \hline
\end{tabular}

${ }^{\mathrm{a}}$ Here and below in Table $\mathrm{V}$ for $\sigma_{1 T}=0$ and Table VII for $\sigma_{1 C} / C=0$ should not be 0.47 but 0.48 . This is because without any tolerance of these variables, in IVA mode, the shape of the output module pulse should be exactly the same as of each cavity. In our simulations the 0.47 instead of 0.48 indicates the simulation error.

because the output pulses of separate cavities are averaged by the aggregate of 30 cavities connected in series in the module.

\section{B. Influence of the switch jitter on the flatness of the output pulse}

The influence of the switch jitter on the flatness of the output pulse is shown in Table IV for one cavity loaded into $R=0.79 \mathrm{Ohm}$ load, and in Table $\mathrm{V}$ for the module of 30 cavities loaded into Rload $=23.7 \mathrm{Ohm}$ load. The value of the capacitances and the inductance of the bricks were kept fixed and equal to the nominal values.

The switch jitter also affects the flatness of the top of the output pulse of the module considerably less than it affects the flatness of the top of the cavity pulse.

\section{Influence of the tolerance of the capacitor capacitance on the flatness of the output pulse}

The influence of the capacitance tolerance on the flatness of the output pulse was investigated at nominal inductance of all cavity bricks $\bar{L}=200 \mathrm{nH}$ and without any spread in the switch closing. The tolerance of the capacitance (in relative units) was assumed to be independent of its nominal value. Table VI presents the results obtained for one cavity loaded into $R=0.79 \mathrm{Ohm}$ load, and Table VII presents the results obtained for the module of 30 series cavities loaded into Rload $=23.7 \mathrm{Ohm}$ load.

TABLE IV. Flatness of the top of the output pulse of the LTD cavity depending on the switch jitter.

\begin{tabular}{llllllc}
\hline \hline$\sigma_{1 T}, \mathrm{~ns}$ & 0 & 0.5 & 1.0 & 1.5 & 2.0 & 2.5 \\
$\mathrm{MDU} / 100 \mathrm{kV}, \%$ & 0.48 & 2.57 & 4.76 & 6.88 & 8.91 & 10.83 \\
\hline \hline
\end{tabular}

TABLE V. Flatness of the top of the output pulse of the LTD module consisting of 30 cavities connected in series depending on the switch jitter.

\begin{tabular}{llllllll}
\hline \hline$\sigma_{1 T}, \mathrm{~ns}$ & 0 & 0.5 & 0.59 & 1.0 & 1.5 & 2.0 & 2.5 \\
$\mathrm{MDU} / 3 \mathrm{MV}, \%$ & 0.47 & 0.902 & 1 & 1.442 & 1.95 & 2.446 & 3.00 \\
\hline \hline
\end{tabular}

TABLE VI. Flatness of the top of the output pulse of the LTD cavity depending on the tolerance of the capacitor capacitance.

\begin{tabular}{llllll}
\hline \hline$\sigma_{1 C} / \bar{C}, \%$ & 0 & 0.833 & 1.67 & 2.5 & 3.33 \\
$\mathrm{MDU} / 100 \mathrm{kV}, \%$ & 0.48 & 0.88 & 1.45 & 2.01 & 2.57 \\
\hline \hline
\end{tabular}

TABLE VII. Flatness of the top of the output pulse of the LTD module consisting of 30 cavities connected in series depending on the tolerance of the capacitor capacitance.

\begin{tabular}{llllllll}
\hline \hline$\sigma_{1 C} / \bar{C}, \%$ & 0 & 0.833 & 1.67 & 2.5 & 3.33 & 6.58 & 6.67 \\
$\mathrm{MDU} / 3 \mathrm{MV}, \%$ & 0.47 & 0.53 & 0.587 & 0.65 & 0.715 & 1 & 1.008 \\
\hline \hline
\end{tabular}

The tolerance of the capacitor capacitance affects the flatness of the top of the output pulse less than the tolerance of the brick inductance. This is because each brick includes two capacitors connected in series, and only their equivalent capacitance is important for the shape of the output pulse.

The capacitors could be selected to have the required capacitance before they are installed into the cavities; then the tolerance of the equivalent capacitance of two series capacitors in each brick can be reduced to almost zero. Such preliminary sorting will increase the cost of the capacitors, but it is much simpler and less expensive than to control the brick inductance or create reliable switches

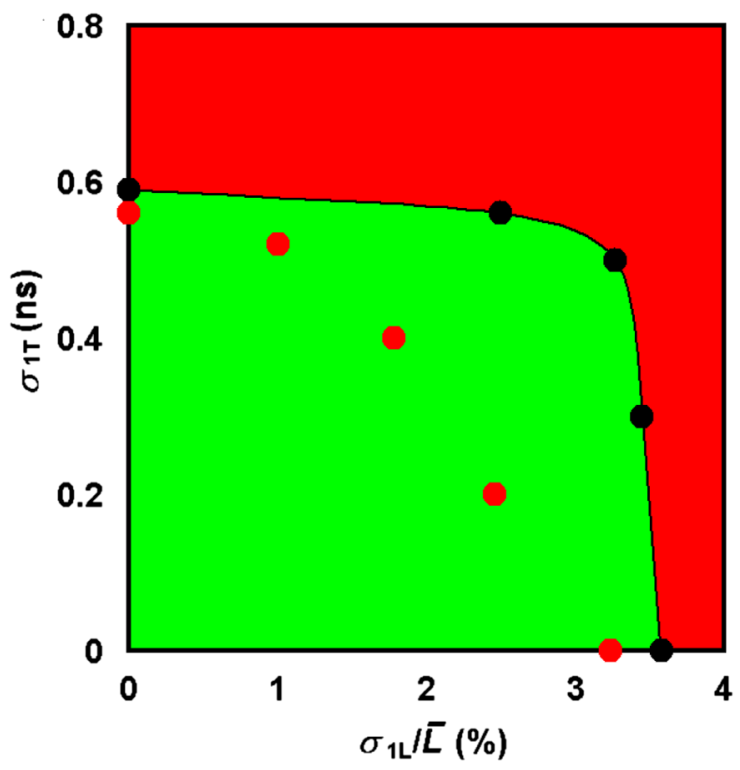

FIG. 8. With green and red the regions are shown where the top of the pulse at the output of the LTD module has larger variation (red) or smaller (green) than $\pm 0.5 \%$ for $\sigma_{1 C} / \bar{C}=0$. On the curve separating these two regions, the top of the output pulse is $\pm 0.5 \%$ flat. Red points on the green section of this graph indicate how the red region would increase (and the green decrease) if the tolerance of the capacitor capacitance $\sigma_{1 C} / \bar{C}=3.33 \%$ is included in the simulations. 

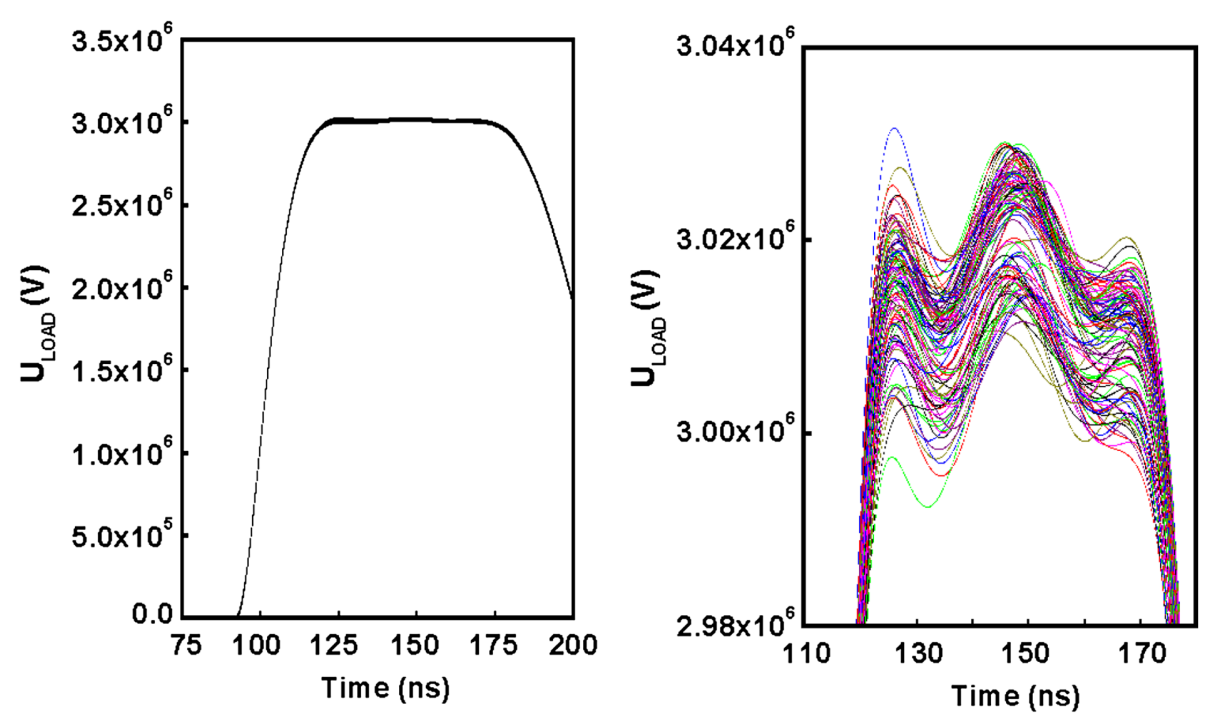

FIG. 9. The left graph shows the output pulses across the $23.7 \mathrm{Ohm}$ load of the module consisting of 30 LTD cavities connected in series with 5 th harmonics in $11 \mathrm{~S}+7 \mathrm{M}+2 \mathrm{~F}$ configuration, which are simulated with $\sigma_{1 T}=0.5 \mathrm{~ns}, \sigma_{1 L} / \bar{L}=1.67 \%, \sigma_{1 C} / \bar{C}=0$. The right graph shows the tops of 80 pulses in more detail.

with negligible jitter. To demonstrate the effect of such sorting, in Subsec. IV D we consider two options for the brick capacitance values: one where the values are fixed and exactly equal to the required for each brick value $\left(\sigma_{1 C} / \bar{C}=0\right)$, and another where the capacitance is allowed to vary according the standard deviation $\left(\sigma_{1 C} / \bar{C}=3.33 \%\right)$. The possibility to get a $\pm 0.5 \%$ voltage flat pulse at the output of the LTD module was evaluated in both cases by using, in addition to the capacitance variance, the tolerance of the brick inductance and the switch jitter.

\section{Joint influence of inductance and switch jitter tolerances on the LTD output pulse flatness}

The graph in Fig. 8 illustrates the joint influence of the tolerance of the brick inductance and the switch jitter at $\sigma_{1 C} / \bar{C}=0$ on the flatness of the output pulse of the module consisting of 30 LTD cavities with 5th harmonics connected in series. This graph has two regions, the red and the green, that are separated by the curve which indicates the values of $\sigma_{1 L} / \bar{L}$ and $\sigma_{1 T}$ providing the $\pm 0.5 \%$ flat output pulse across the $23.7 \mathrm{Ohm}$ matched load. In the green region the top of the module pulse has a smaller than $\pm 0.5 \%$ variation, while in the red it has larger than $\pm 0.5 \%$ variation.

Red points in the green region of the graph indicate how the red region would increase (and the green decrease) if the tolerance of the capacitor capacitance becomes $\sigma_{1 C} / \bar{C}=3.33 \%$.

On the left of Fig. 9 are shown the output pulses across the $23.7 \mathrm{Ohm}$ resistive load of the module consisting of 30 LTD cavities connected in series with 5 th harmonics in $11 \mathrm{~S}+7 \mathrm{M}+2 \mathrm{~F}$ configuration. These results were obtained assuming $\sigma_{1 T}=0.5 \mathrm{~ns}, \sigma_{1 L} / \bar{L}=1.67 \%$, and $\sigma_{1 C} / \bar{C}=0$.
These values of $\sigma_{1 T}$ and $\sigma_{1 L} / \bar{L}$, located in the green region of Fig. 8, provide a pulse flat top with less than $\pm 0.5 \%$ variation value. For all these pulses the rise time measured between $10 \%$ and $90 \%$ of $U_{\max }=3 \mathrm{MV}$ is $\sim 16.8 \mathrm{~ns}$, the length of the top at $90 \%$ of $U_{\max }$ is $\sim 75 \mathrm{~ns}$. The efficiency of energy transfer from such LTD module into the 23.7 Ohm load is expected to be $\sim 80 \%$. On the right of Fig. 9 an overlay of 80 top sections of these pulses are shown in more detail.

\section{CONCLUSION}

We have investigated the accuracy of the capacitor capacitance, the brick inductance, and the switch closing jitter influence on the flatness of the output pulse module consisting of 30 LTD cavities with 5th harmonics connected in series and operating in IVA mode. The preferable module cavity configuration is $11 \mathrm{~S}+7 \mathrm{M}+2 \mathrm{~F}$ and has as a load a Rload $=23.7 \mathrm{Ohm}$.

Statistical simulation of such module behavior was performed using the OrCAD software, which is based on the PSPICE circuit code. All the above-mentioned statistically varying parameters were assumed to obey Gaussian distribution. The nominal values of the capacitor capacitance in the bricks generating the 1st, 3rd, and 5th harmonics were assumed to be $12 \mathrm{nF}, 1.4 \mathrm{nF}$, and $0.5 \mathrm{nF}$, respectively. The nominal value of the inductance of each brick was assumed to be $200 \mathrm{nH}$. The nominal delay between the switch closing of two neighboring LTD cavities was assumed to be $\tau_{i}=0.73$ ns. This delay is equal to the electrical length of one cavity along its vacuum insulated uniform coaxial section of the output line. Since the capacitor capacitance can be selected before the capacitors are installed into the cavities, the capacitance 
tolerance can be assumed equal to zero. Our simulations show that this would allow us to get the $\pm 0.5 \%$ flat output pulse top if the switch jitter $\sigma_{1 T}$ and the tolerance of the brick inductance $\sigma_{1 L} / \bar{L}$ are on the curve separating the green and red regions in Fig. 8 .

One important feature observed in this work is the favorable influence of the large number of cavities connected in series into the module on the flatness of its output pulse. Simulations show that, although for the presently achievable $\sigma_{1 T} \approx 2 \mathrm{~ns}$ and $\sigma_{1 C} / \bar{C} \approx \sigma_{1 L} / \bar{L} \approx 3.33 \%$ the flatness of the cavity pulse is expected to be $\sim \pm 6 \%$, it improves to $\sim \pm 1.5 \%$ at the output pulse of the module consisting of 30 such cavities connected in series. This is very interesting and promising that further increasing the number of cavities in a module will result in a higher degree of flatness of the output pulse.

\section{ACKNOWLEDGMENTS}

Sandia is a multi-program laboratory managed and operated by Sandia Corporation, a wholly owned subsidiary of Lockheed Martin Company, for the United States Department of Energy's National Nuclear Security Administration under Contract No. DE-AC04-94AL85000.
[1] A. A. Kim, M. G. Mazarakis, V. M. Alexeenko, S. S. Kondratiev, J. J. Leckby, and B. V. Oliver, Square pulse LTD with 5th harmonic bricks, in Proceedings of the 19th IEEE International Pulsed Power Conference, San Francisco, California, USA, June 16-21, 2013, edited by B. Oliver and M. Crowford (IEEE, Piscataway, NJ, 2013), pp. 1354-1356.

[2] OrCAD Capture CIS 10.5.

[3] http://www.metglas.com/products/magnetic_materials/2605s3a .asp.

[4] A. A. Kim, M. G. Mazarakis, V. I. Manylov, V. A. Vizir, and W. A. Stygar, Energy loss due to eddy current in linear transformer driver cores, Phys. Rev. ST Accel. Beams 13, 070401 (2010).

[5] L. Peng, S. Fengju et al., Influences of switching jitter on the operational performances of linear transformer driversbased drivers, Plasma Sci. Technol. 14, 347 (2012).

[6] V. M. Alexeenko, M. G. Mazarakis et al., Sensitivity studies of the switch jitter effect on the Square pulse LTD with three harmonics, Izvestia Vuzov. Physica 57, 5 (2014); Proceedings of 18th Symposium on High Current Electronics, Tomsk, Russia.

[7] A. A. Kim, V. A. Sinebryukhov et al., Pulse shaping in Square pulse LTD, IzvestiaVuzov. Physica 55, 176 (2012); Proceedings of 17th Symposium on High Current Electronics, Tomsk, Russia. 